\title{
Colorectal cancer genomic biomarkers in the clinical management of patients with metastatic colorectal carcinoma
}

Anna Maria Rachiglio, Alessandra Sacco, Laura Forgione, Claudia Esposito, Nicoletta Chicchinelli, Nicola Normanno* ${ }^{*}$

Cell Biology and Biotherapy Unit, Istituto Nazionale Tumori-IRCCS-Fondazione G. Pascale, 80131 Naples, Italy

*Correspondence: Nicola Normanno, Cell Biology and Biotherapy Unit, Istituto Nazionale Tumori-IRCCS-Fondazione G. Pascale, 80131 Naples, Italy. n.normanno@istitutotumori.na.it

Academic Editor: Graham Packham, University of Southampton, UK

Received: January 16, 2020 Accepted: February 12, 2020 Published: February 29, 2020

Cite this article: Rachiglio AM, Sacco A, Forgione L, Esposito C, Chicchinelli N, Normanno N. Colorectal cancer genomic biomarkers in the clinical management of patients with metastatic colorectal carcinoma. Explor Target Antitumor Ther. 2020;1:53-70. https://doi.org/10.37349/etat.2020.00004

\begin{abstract}
Colorectal carcinoma (CRC) is an heterogeneous disease in which different genetic alterations play a role in its pathogenesis and progression and offer potential for therapeutic intervention. The research on predictive biomarkers in metastatic CRC (mCRC) mainly focused on the identification of biomarkers of response or resistance to anti-epidermal growth factor receptor monoclonal antibodies. In this respect, international guidelines suggest testing mCRC patients only for KRAS, NRAS and BRAF mutations and for microsatellite instability. However, the use of novel testing methods is raising relevant issue related to these biomarkers, such as the presence of sub-clonal RAS mutations or the clinical interpretation of rare noV600 BRAF variants. In addition, a number of novel biomarkers is emerging from recent studies including amplification of ERBB2, mutations in ERBB2, MAP2K1 and NF1 and rearrangements of ALK, ROS1, NTRK and RET. Mutations in POLE and the levels of tumor mutation burden also appear as possible biomarkers of response to immunotherapy in CRC. Finally, the consensus molecular subtypes classification of CRC based on gene expression profiling has prognostic and predictive implications. Integration of all these information will be likely necessary in the next future in order to improve precision/personalized medicine in mCRC patients.
\end{abstract}

\section{Keywords}

Colorectal carcinoma, molecular biomarker, precision medicine

\section{Introduction}

Colorectal cancer (CRC) is the third most common cancer in men and the second most common in women [1]. Although screening programs have significantly improved the early diagnosis of CRC, a significant fraction of patients either have metastatic lesions at diagnosis or will develop metastases during follow up. As such, a significant part of cancer research has focused on identifying novel therapies in the field of metastatic CRC (mCRC) [2]. 


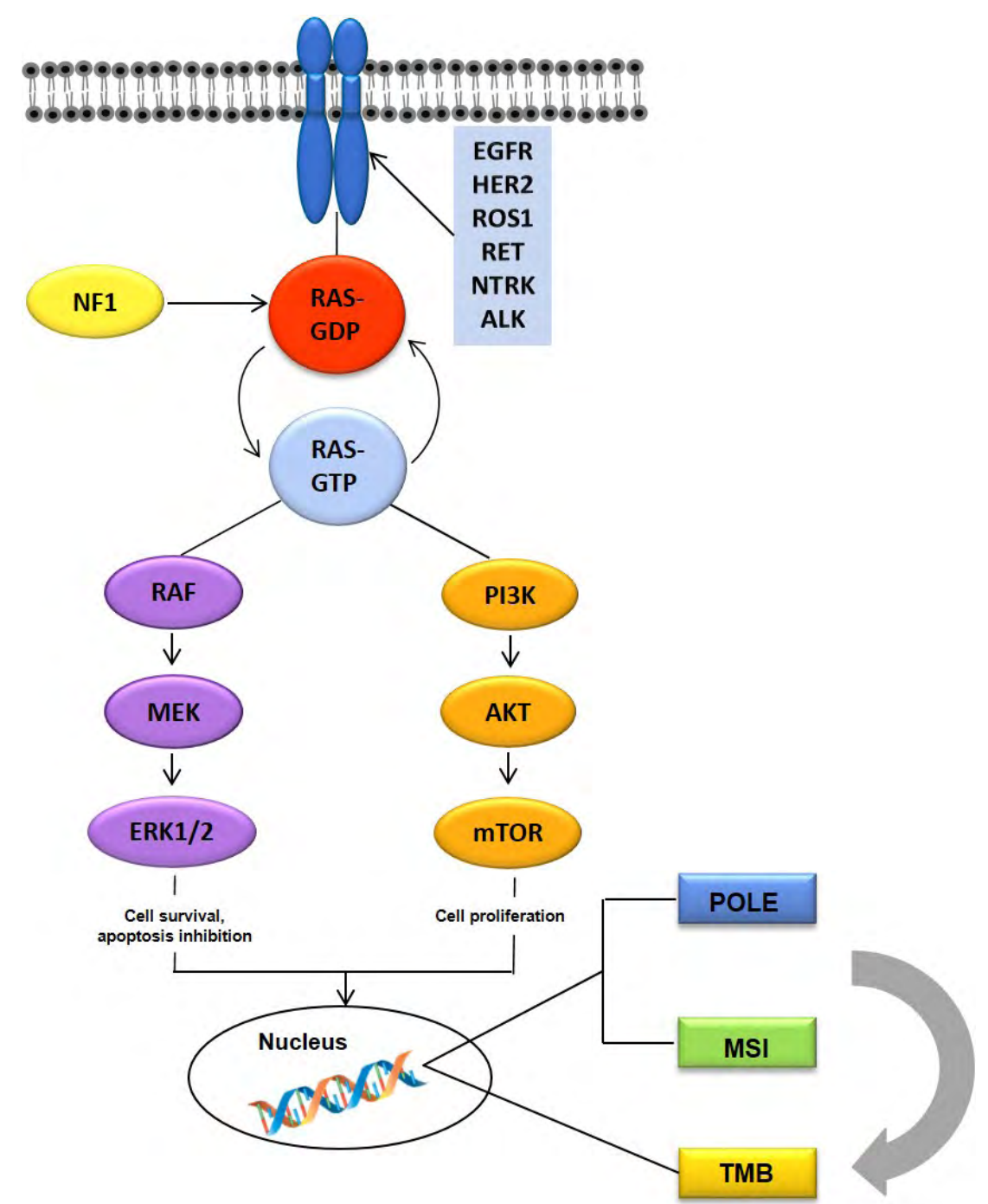

Figure 1. Genomic predictive or prognostic alterations in colorectal cancer

The availability of highly active chemotherapeutics and the approval of several targeted therapies, such as agents directed against the epidermal growth factor receptor (EGFR) and the vascular endothelial growth factor (VEGF) and its receptors, led to significant improvements in tumor response rates and survival in patients with mCRC [3]. However, despite the multitude of treatments available, outcomes and toxicity with each regimen can vary markedly from patient to patient [4]. The research on predictive biomarkers in mCRC mainly focused on the identification of biomarkers of response or resistance to anti-EGFR monoclonal antibodies [5]. However, CRC is a highly heterogeneous disease in which different signaling pathways contribute to cancer pathogenesis and progression [6]. Genetic alterations involved in the resistance to EGFR monoclonal antibodies might also offer opportunities for therapeutic intervention. Therefore, comprehensive genomic profiling might significantly improve the use of personalized therapy in patients with MCRC.

In this review article, we will briefly discuss the predictive biomarkers that have been currently approved by guidelines for the management of mCRC patients and their impact on treatment decision making, and we will next summarize current evidence on emerging biomarkers that might improve precision/personalized therapy in mCRC (Figure 1).

\section{Current biomarkers}

The guidelines of the European Society of Medical Oncology (ESMO) indicate as mandatory for the management of mCRC the testing of KRAS, NRAS and BRAF mutations and the assessment of the microsatellite instability (MSI) status [4] (Table 1). 
Table 1. Summary of current and emerging molecular biomarkers associated with targeted therapies

\begin{tabular}{|c|c|c|c|}
\hline \multicolumn{2}{|l|}{ Biomarker } & Agents & Status \\
\hline \multicolumn{2}{|l|}{ KRAS/NRAS } & $\begin{array}{l}\text { Cetuximab, Panitumumab } \\
\text { (negative predictive biomarker) }\end{array}$ & Approved EMA and FDA \\
\hline \multicolumn{4}{|l|}{$B R A F$} \\
\hline & V600E & $\begin{array}{l}\text { Encorafenib + Binimetinib } \\
+ \text { Cetuximab [57] }\end{array}$ & Approved FDA \\
\hline & nonV600E & $\mathrm{MEK} \pm \mathrm{BRAF} \pm \mathrm{EGFR}$ inhibitors & Experimental \\
\hline MSI & & $\begin{array}{l}\text { Pembrolizumab }[66,67] \\
\text { Nivolumab [68] }\end{array}$ & Approved FDA \\
\hline HER2 & & $\begin{array}{l}\text { Trastuzumab + Pertuzumab [84]; } \\
\text { Lapatinib + Trastuzumab [85] }\end{array}$ & Experimental \\
\hline NTRK & & Larotrectinib, Entrectinib & $\begin{array}{l}\text { Approved EMA (Larotrectinib) and FDA } \\
\text { (Larotrectinib, Entrectinib) }\end{array}$ \\
\hline$N F 1$ & & $\mathrm{MEK} \pm \mathrm{BRAF} \pm \mathrm{EGFR}$ inhibitors & Experimental \\
\hline
\end{tabular}

\section{RAS}

RAS is a family of GTPase proteins whose signaling regulates key processes in tumor pathogenesis, including cell proliferation, differentiation, adhesion, migration and survival [7]. Activating mutations of $R A S$ genes are indeed involved in the progression of different tumor types. The three RAS genes (HRAS, NRAS, and KRAS) still represent the most mutated oncogene family in cancer (30\%) and an estimated $52 \%$ of CRCs carry mutations in any RAS gene. KRAS is the most frequently mutated gene among the RAS family, representing $86 \%$ of RAS mutations in CRC, followed by $14 \%$ NRAS mutations [8]. KRAS mutations occur in approximately $44 \%$ of CRC, and the majority affect codons 12 (30\%) and $13(8 \%)$ of exon 2 . An additional $6 \%$ of mutations are found in KRAS exons 3 and 4, while 5\% of mutations are in NRAS exons 2, 3 or 4 [9].

RAS proteins are essential components of the EGFR signaling cascade and, therefore, their constitutive activation might lead to resistance to anti-EGFR monoclonal antibodies [5]. Indeed, a series of retrospective analyses has shown that KRAS exon 2 mutations (codons 12 and 13) are associated with resistance to antiEGFR therapy in patients with $\mathrm{mCRC}$, in the context of randomized studies in which the anti-EGFR cetuximab or panitumumab monoclonal antibodies were used alone or in combination with chemotherapy [10-17] (Table 2). Subsequently, activating KRAS hotspot mutations in exons 3 and 4 and in exons 2, 3 and 4 of NRAS were found to also predict for lack of benefit from anti-EGFR therapies, refining the population of patients who might benefit these agents [18-23] (Table 2).

Testing for RAS mutational status is currently recommended for all patients at the time of mCRC diagnosis $[4,24]$. The introduction in clinical molecular diagnostics of novel techniques with high sensitivity raised the question of the threshold of $R A S$ mutations to identify patients that are truly resistant to antiEGFR agents. Although RAS mutations are an early event in CRC pathogenesis in most cases, a fraction of CRC carrying sub-clonal RAS mutations has been identified $[25,26]$. The occurrence of sub-clonal KRAS mutations at low allelic frequency has been confirmed in studies that explored the use of liquid biopsy for KRAS mutation testing in mCRC [27, 28]. Recent publications suggested that a 5\% cutoff should be the optimal threshold for the detection of $R A S$ mutations in the clinical scenario [29, 30].

Finally, RAS mutations have been investigated in CRC only as marker of resistance to anti-EGFR agents up to now because of the lack of active agents targeting RAS proteins. However, promising preliminary results have been obtained with novel drugs targeting RAS mutations [31]. These findings are opening new therapeutic opportunities for patients carrying RAS mutant CRC (Table 2).

In particular, different drugs targeting the KRASG12C mutation are in clinical development in patients with solid tumors, including CRC. In a recent study, Hallin et al. [32], showed that MRTX849 is a selective and covalent KRASG12C inhibitor. Indeed, this small molecule caused tumor shrinkage in 65\% of KRASG12C positive cell line-derived and patient-derived xenograft models of multiple solid tumor types. Furthermore, objective responses have been observed in KRASG12C-positive lung and colon adenocarcinoma patients treated with MRTX849 [32]. In an ongoing phase I/II trial responses to this drug have been observed in both lung and CRC KRASG12C mutant patients [33] (Table 2).

Another novel small molecule that specifically and irreversibly inhibits KRASG12C by locking it in an 
inactive GDP-bound state is AMG 510 [34]. A phase 1 open-label, multicenter study is underway to evaluate the safety, tolerability, pharmacokinetics, and efficacy of AMG 510 in patients with locally-advanced/ metastatic KRASG12C solid tumors and the preliminary data suggest the antitumor-activity of this agent in different tumor types, including CRC [35] (Table 2).

Table 2. Main clinical trials that explored predictive biomarkers in CRC

\begin{tabular}{|c|c|c|}
\hline Biomarker & Clinical trial & Results \\
\hline \multirow[t]{7}{*}{$R A S$} & $\begin{array}{l}\text { PRIME } \\
{[15]}\end{array}$ & $\begin{array}{l}\text { The combination of panitumumab and FOLFOX4 significantly improved PFS in } \\
\text { patients with KRAS wild-type tumors. }\end{array}$ \\
\hline & $\begin{array}{l}\text { CRYSTAL } \\
{[20]}\end{array}$ & $\begin{array}{l}\text { Molecular testing of tumors for all activating RAS mutations is essential before } \\
\text { considering anti-epidermal growth factor receptor therapy, thereby allowing the } \\
\text { further tailoring of cetuximab administration to maximize patient benefit. }\end{array}$ \\
\hline & $\begin{array}{l}\text { OPUS } \\
{[16]}\end{array}$ & $\begin{array}{l}\text { The addition of cetuximab to FOLFOX-4 significantly improved PFS and response } \\
\text { in patients with KRAS wild-type tumors. }\end{array}$ \\
\hline & $\begin{array}{l}\text { MRC COIN } \\
{[17]}\end{array}$ & $\begin{array}{l}\text { This trial has not confirmed a benefit of addition of cetuximab to oxaliplatin-based } \\
\text { chemotherapy in first-line treatment of patients with advanced CRC. }\end{array}$ \\
\hline & $\begin{array}{l}\text { FIRE-3 } \\
{[23]}\end{array}$ & $\begin{array}{l}\text { In this study a significantly higher OS was demonstrated for FOLFIRI plus } \\
\text { cetuximab compared to FOLFIRI plus bevacizumab in the extended RAS wild- } \\
\text { type subgroup. }\end{array}$ \\
\hline & $\begin{array}{l}\text { NCT03600883 } \\
{[35]}\end{array}$ & $\begin{array}{l}\text { Early results suggest antitumor activity of single-agent AMG } 510 \text { in KRAS }{ }^{\mathrm{G} 12 \mathrm{C}} \\
\text { mutant solid tumors. }\end{array}$ \\
\hline & $\begin{array}{l}\text { NCT03785249 } \\
{[33]}\end{array}$ & $\begin{array}{l}\text { The trial is ongoing and responses to treatment with MRTX849 have been } \\
\text { observed in both lung and CRC KRASG12C mutant patients. }\end{array}$ \\
\hline \multirow[t]{2}{*}{$B R A F$} & $\begin{array}{l}\text { SWOG } 1406 \\
{[62]}\end{array}$ & $\begin{array}{l}\text { The addition of vemurafenib to the combination of cetuximab and irinotecan } \\
\text { in BRAFV } 600 \text { mutated tumors resulted in a prolongation of PFS and a higher } \\
\text { disease control rate. }\end{array}$ \\
\hline & $\begin{array}{l}\text { BEACON CRC } \\
{[63]}\end{array}$ & $\begin{array}{l}\text { A combination of encorafenib, cetuximab, and binimetinib resulted in significantly } \\
\text { longer OS and a higher response rate than standard therapy in patients with } \\
\text { mCRC with the BRAF V } 600 \text { E mutation. }\end{array}$ \\
\hline \multirow[t]{2}{*}{ MSI } & $\begin{array}{l}\text { NCT01876511 } \\
{[71]}\end{array}$ & $\begin{array}{l}\text { This study showed that mismatch-repair status predicted clinical benefit of } \\
\text { immune checkpoint blockade with pembrolizumab. }\end{array}$ \\
\hline & $\begin{array}{l}\text { CheckMate } 142 \\
{[73]}\end{array}$ & $\begin{array}{l}\text { Nivolumab provided durable responses and disease control in pre-treated } \\
\text { patients with dMMR/MSI-H mCRC. }\end{array}$ \\
\hline \multirow[t]{2}{*}{ HER-2 } & $\begin{array}{l}\text { HERACLES } \\
{[81]}\end{array}$ & $\begin{array}{l}\text { The combination of trastuzumab and lapatinib is active and well tolerated in } \\
\text { treatment-refractory patients with HER2-positive tumors. }\end{array}$ \\
\hline & $\begin{array}{l}\text { MyPathway } \\
\text { [86] }\end{array}$ & $\begin{array}{l}\text { Durable responses were seen in patients with refractory colorectal cancers with } \\
\text { HER2 activation/overexpression when the approved targeted therapy regimen } \\
\text { administered without chemotherapy. }\end{array}$ \\
\hline \multirow[t]{2}{*}{ CMSs } & $\begin{array}{l}\text { CALGB/SWOG } 80405 \\
{[131]}\end{array}$ & The CMSs are highly prognostic and predictive for OS and PFS. \\
\hline & $\begin{array}{l}\text { Fire-3(AIO KRK-0306) } \\
{[132]}\end{array}$ & $\begin{array}{l}\text { Prolonged OS induced by FOLFIRI plus cetuximab versus FOLFIRI plus } \\
\text { bevacizumab in the FIRE- } 3 \text { study appears to be driven by CMS } 2 \text { and CMS } 4 \text {. }\end{array}$ \\
\hline
\end{tabular}

OS: overall survival

\section{BRAF}

Approximately $8 \%$ of advanced CRC cases carry activating mutations of $B R A F$, a key protein in the mitogenactivated protein-kinase (MAPK) signaling pathway [36, 37]. Roughly $90 \%$ of $B R A F$ mutations in CRC occur as a T1799 transversion in exon 15, which leads to the substitution of valine for glutamic acid (V600E) [38]. This substitution regulates phosphorylation, increasing BRAF activity by approximately ten times compared to the wild-type protein [39]. Of note, patients carrying BRAF V600E mutations have distinct clinical and pathological features including right sided tumors, high grade, older age, female sex, T4 tumors, mucinous histology, poorly differentiated tumors and microsatellite instability [40-42]. Nevertheless, BRAF mutations can also be detected in left-sided tumors [43].

The BRAF V600E mutation is a strong negative prognostic biomarker in $\mathrm{mCRC}$, as demonstrated in several clinical trials and metanalyses [13, 21, 44-46]. In patients with disease recurrence after primary tumor resection, the presence of the $B R A F \mathrm{~V} 600 \mathrm{E}$ mutation is associated with reduced post-recurrence survival [47]. Similarly, some studies have shown that in patients receiving liver metastasis resection, the $B R A F$ V600E mutation is correlated with shorter survival [48-50]. However, approximately $25 \%$ of patients with $B R A F$ V600E mutations have a good outcome, similar to no-BRAF mutant cases, thus suggesting that the BRAF V600E population of CRC patients is heterogeneous [51]. The BRAF V600 mutations are classified 
as class I mutations leading to RAS-independent high level of activation of BRAF, based on functional studies on non-CRC preclinical models [52, 53]. In contrast, class II mutations (affecting codons 597 and 601) induce intermerdiate, RAS-independent BRAF activation, while class III variants (in codons 594 and 596) are RAS-dependent and produce a low level of BRAF activation. Alterations in class II or III are less frequent as compared to class I and account for $2.2 \%$ of all patients tested or $21.6 \%$ of all $B R A F$ mutations in CRC [54]. Recent data suggest that class III $B R A F$ mutations are associated with a favorable prognosis in patients with mCRC, while class II mutant cases seem to have an outcome similar to patients with class I BRAF mutations [54-56]. However, the limited number of cases included in these studies suggests that additional experimentation should be performed in this field.

While the negative prognostic role of the $B R A F \mathrm{~V} 600 \mathrm{E}$ mutations is quite clear, its predictive role as a mechanism of resistance to anti-EGFR monoclonal antibodies-based therapy in the first-line treatment of mCRC patients is less established. In particular, two meta-analyses on the predictive role of the BRAF V600E mutation for anti-EGFR agents came to completely different conclusions, although analyzing the same clinical trials. Pietrantonio et al. [57], concluded that the BRAF V600E mutations are a negative predictive factor for anti-EGFR agents, thus supporting the exclusion of patients carrying such genetic alterations from treatment with these agents. In contrast, another meta-analysis by Rowland et al. [58], concluded that the evidence is insufficient in order to justify the exclusion of anti-EGFR agents in the case of patients with $B R A F$ V600E mutations. Importantly, pre-clinical data suggest that patients with class III mutations may be sensitive to EGFR inhibitors [53].

The poor prognosis of $B R A F$ V600 mutant mCRC patients led to the exploration of novel therapeutic approaches. Unlike what occurrs in melanoma, mCRC patients with BRAF V600E mutation do not respond to BRAF inhibitors. This phenomenon is due to a specific molecular mechanism demonstrated in CRC cells that involves the activation of alternative signal transduction pathways through the EGFR [59]. This last observation led to explore the activity of drug combinations containing BRAF, MEK and/or PI3K inhibitors together with anti-EGFR monoclonal antibodies. An open-label phase I/II study in patients with BRAF V600E mutation-positive $\mathrm{mCRC}$, demonstrated that patients receiving triple therapy (dabrafenib, trametinib and panitumumab) had a numerically improved objective response rate (ORR) (21\%) compared with those receiving panitumumab plus either dabrafenib (10\%) or trametinib (0\%) and had a longer progression free survival (PFS) (4.2 vs 3.5 vs 2.6 months) [60, 61]. Moreover, in a randomized trial, addition of vemurafenib to the combination of cetuximab and irinotecan resulted in prolonged PFS [4.4 vs 2.0 months, hazard ratio (HR) $0.42,95 \%$ confidence interval (CI) $0.26-0.66, P<0.001$ ] and a higher disease control rate $(67 \%$ vs $22 \% ; P<0.001)$ compared with cetuximab and irinotecan treatment alone in heavily pre-treated patients with BRAF-mutant mCRC [62] (Table 2).

The combination of encorafenib (BRAF inhibitor), binimetinib (MEK inhibitor) and cetuximab is being assessed in the BEACON trial in BRAF-mutant mCRC patients who had disease progression after one or two previous regimens. Patients are randomly assigned to receive encorafenib, binimetinib, and cetuximab (triplet-therapy group); encorafenib and cetuximab (doublet-therapy group); or the investigators' choice of either cetuximab and irinotecan or cetuximab and FOLFIRI (control group). The results of a pre-specified interim analysis of the BEACON trial showed a median overall survival of 9.0 months in the triplet-therapy group, 8.4 months in the doublet-therapy group and 5.4 months in the control group [63] (Table 2). Based on these data, the Food and Drug Administration (FDA) approved such combination for BRAF V600 mutant mCRC patients (Table 1).

\section{Microsatellite instability}

Microsatellites are repeated DNA sequences of 1-6 bp that can be found in coding and noncoding regions of the genome. Microsatellite instability (MSI) results from inactivation of the mismatch repair (MMR) genes through sporadic $M L H 1$ promoter hypermethylation and/or germline/somatic mutations in MMR genes, such as MLH1, MSH2, MSH6, or PMS2 that are also involved in the Lynch syndrome [64, 65]. MMR deficiency leads to the accumulation of somatic mutations and induces genomic instability, causing cancer-associated 
alterations [66]. MSI is detected in approximately $15 \%$ of patients with mCRC; only $3 \%$ of the cases are associated with Lynch syndrome and the other $12 \%$ are caused by sporadic hyper-methylation of the MLH1 gene and/or germline/somatic mutations in MMR genes [67].

MMR/MSI status testing is recommended for patients with CRC for prognostic stratification [24]. Based on the number of markers altered, CRC are classified in MSI-high (MSI-H), MSI-low (MSI-L) and microsatellite stable (MSS) if the test is performed on DNA, and in MMR-proficient (pMMR) or deficient (dMMR) if the MMR proteins are tested with immunohistochemistry. In clinical practice, the recommended method for MSI/MMR testing is immunohistochemistry. Alternatively, testing can be performed by polymerase chain reaction (PCR)-based assessment of microsatellite alterations using five microsatellite markers, including at least BAT-25 and BAT-26. Finally, next-generation sequencing (NGS), coupling MSI and tumor mutation burden (TMB) analysis with comprehensive genomic profiling, may represent a more adequate tool for selecting patients for immunotherapy, for common or rare cancers not belonging to the spectrum of Lynch syndrome [68].

A number of different studies have demonstrated that early stage MSI-H CRC patients have better prognosis as compared with MSI-L and MSS patients [69]. As a consequence of the good prognosis of MSI-H tumors, this biomarker is positive in only about $4 \%$ of mCRC. However, the prognostic significance of MSI in the advanced stages of the disease is different from the initial ones. In fact, in mCRC the presence of MSI is associated with a worse prognosis as compared with MSS [44].

MMR deficient tumors have a high mutational load that can produce multiple immunogenic neoantigens, which will increase the probability of response to immune checkpoint inhibitors [70]. In this regard, several phase II clinical trials demonstrated a high rate of responses to PD-1/PD-L1 inhibitors in patients with mCRC and MSI-H or dMMR [71-73] (Table 2). Interestingly, responses have been obtained in MSI patients with different histological types of cancer [72]. Based on these data, the FDA with a historic decision approved the use of pembrolizumab in patients with MSI regardless of the histological origin of the tumor (Table 1).

Different studies confirmed that MSI CRC has a higher TMB as compared with MMS tumors [74, 75]. In particular, a very good correlation between MSI-status and TMB levels was found in CRC [64]. However, a wide range of TMB value have been found also among MSI CRC. In this respect, patients with MSI CRC and high TMB showed and higher ORR and a longer PFS and OS as compared with MSI CRC patients with lower TMB. Therefore, TMB appears to be an important independent biomarker within MSI-H mCRC to stratify patients for likelihood of response to immune checkpoint inhibitors [75].

\section{Emerging biomarkers}

HER2 amplification and mutations

The HER2 oncogene is coded by the ERBB2 gene and belongs to the EGFR family of tyrosine kinase receptors that includes also EGFR, HER-3 and HER-4.

Recent studies have highlighted a possible role of genetic alterations of HER2 in the pathogenesis of CRC. Amplification or mutations of ERBB2, both point and small insertions/deletions, have been described in a subset of patients with CRC [6]. The percentage of cases with ERBB2 alterations varies considerably between studies, probably due to the limited number of cases analyzed and the variety of analysis methods used, especially for gene amplification studied in some cases only at the level of protein expression by immunohistochemistry [76]. A recent study, which analyzed 8,887 cases of mCRC by means of NGS techniques, highlighted the presence of $E R B B 2$ amplification in $2.8 \%$ of cases, mutations in $1.5 \%$ and amplification and mutation in $0.4 \%$ [77]. Since the frequency of $R A S$ mutations is generally lower among cases with ERBB2 amplification than in those without amplification, the frequency of ERBB2 amplification in wild-type RAS tumors is expected in the order of 4-5\% [76, 77]. Overexpression of HER2 correlates in CRC with an aggressive tumor behavior, which includes profound invasion, lymphatic metastases, distant metastases, perineural invasion [78-80]. However, there are conflicting data on the prognostic role of ERBB2 alterations as well as on the association with the disease site [76]. 
Some studies have explored the predictive role of ERBB2 amplification as biomarker of resistance to anti-EGFR drugs in mCRC patients [81-85]. These studies described a reduced response rate and a shorter survival in mCRC patients with ERBB2 amplification compared to those without ERBB2 amplification when treated with anti-EGFR monoclonal antibodies. However, these studies are all retrospective analyses, with heterogeneous cases treated in different lines and with different drug combinations. More importantly, all patients included in these analyses (negative and positive for the amplification of ERBB2) received treatment with anti-EGFR drugs. Therefore, these studies do not allow formally distinguishing between a prognostic or predictive effect of $E R B B 2$ amplification, which should be explored in the context of randomized trials. Finally, the predictive value of $E R B B 2$ amplification may be different in the various treatment lines. Sartore-Bianchi et al. [85], have described a trend of different HR between the I, II and III-IV treatment line for the predictive value of ERBB2 amplification in mCRC patients treated with anti-EGFR drugs, although not statistically significant.

Encouraging preliminary data suggest that $E R B B 2$ amplification could be an important marker of response to anti-ERBB2 drugs. In phase II studies that used different combinations of anti-ERBB2 drugs, response rates ranging between $30 \%$ and $71 \%$ have been described [81, 86-89] (Table 2). In particular, the phase 2 HERACLES study evaluated the efficacy of trastuzumab and lapatinib in 27 HER2-positive/KRAS exon 2 wild-type patients with mCRC who had progressed on all other therapies, including cetuximab [81]. An ORR of $30 \%$ was observed, with additional $44 \%$ of the patients experiencing a stabilization of the disease. The mPFS was 21 weeks. However, the mPFS of patients harboring tumors with high levels of ERBB2 gene copy number ( $\geq 945$ as assessed by real time PCR) was 29 weeks, compared with a mPFS of 16 weeks for patients with tumors scoring below this threshold. These data suggest that a quantitative assessment of ERBB2 amplification is needed to identify patients that are more sensitive to anti-HER2 drugs. In agreement with this hypothesis, a correlation was found between plasma copy number of ERBB2 and activity of trastuzumab plus lapatinib in mCRC patients [90]. In the phase 2 basket study MyPathway, 57 mCRC patients with ERBB2 amplification were treated with trastuzumab plus pertuzumab [89]. One (2\%) patient had a complete response and 17 (30\%) had partial responses, with an ORR of 32\%. In this heavily pre-treated cohort of patients (a median of four previous treatment regimens), mPFS was 2.9 months and estimated middle overall survival (mOS) was 11.5 months. An exploratory analysis revealed that 13/56 tumors tested had cooccurring KRAS mutations. In a post-hoc exploratory analysis of survival outcomes by KRAS mutation status, ORR, mPFS and mOS were notably worse in patients with KRAS-mutated tumors as compared with those with KRAS wild-type disease. Numerous studies are underway and their results will be important to better define the role of anti-ERBB2 therapies in mCRC [86, 91].

A pre-clinical study suggested that CRC cells carrying activating mutations of ERBB2 are sensitive to anti-HER2 agents [92]. However, the results of a basket trial in which patients with ERBB2 mutations were treated with the pan-HER inhibitor neratinib showed that MCRC patients did not respond to this therapy unlike other histological types, although carrying similar mutations [93]. While these data must be considered preliminary, this observation raises the question whether ERBB2 mutations are true drivers of tumor growth in CRC (Table 1).

\section{PIK3CA}

The PIK3CA gene encodes the $\mathrm{p} 110 \alpha$ subunit of the phosphatidylinositol-3-kinases (PI3K), which is activated by growth factor stimulation through receptor tyrosine kinases and in turn activates the AKTmTOR signaling pathway [94]. When mutated, PIK3CA induces constitutive phosphorylation of AKT, which promotes cell growth and suppresses apoptosis in cancer cells. [95].

The PIK3CA gene is mutated in many different tumors, including CRC [94]. In particular, mutations of PIK3CA have been described in $15-20 \%$ of CRC, with about $80 \%$ of mutations found in exons 9 and 20 [96-98]. In CRC, PIK3CA mutations are frequently associated with other molecular alterations, including KRAS mutations (especially exon 9 mutations) and high-degree $\mathrm{CpG}$ island methylator phenotype [99].

While PIK3CA mutations seem not to have a prognostic role in CRC [100], their predictive role in both 
early and metastatic CRC has been investigated in several studies.

In patients with early CRC, the presence of PIK3CA mutations was associated with a reduced risk of relapse in patients with regular use of aspirin [101]. In particular, in patients with PIK3CA mutated CRC, the use of aspirin was associated with a reduction of tumor specific and overall mortality of $82 \%$ and $46 \%$, respectively. This effect could be due to the ability of PIK3CA to induce the expression of cyclooxygenase-2 (COX2). However, rofecoxib, a selective COX2 inhibitor, did not show any effect on tumor recurrence in PIK3CA mutant CRC patients, suggesting that other, non-COX2-related mechanisms of action of aspirin might play a relevant role in its protective effect [102].

Several retrospective studies investigated the impact of PIK3CA mutations on the outcome of mCRC receiving anti-EGFR monoclonal antibodies. While some studies suggested a possible role of PIK3CA mutations in the primary resistance to anti-EGFR agents [103-107], other studies failed to confirm such correlation [108].

The discordance between these studies could be due in part to the different mechanisms of action of the exon 9 and exon 20 PIK3CA mutations. Exon 9 mutations interfere with the inhibitory activity of the p85 regulatory subunit and, therefore, require interaction with RAS proteins for activation. In contrast, mutations in exon 20 lead to constitutive activation of PI3K enzymatic activity independently from RAS [94]. In agreement with this hypothesis, in a retrospective study, including $743 \mathrm{mCRC}$ patients receiving antiEGFR therapies, De Roock et al. [98], found that in KRAS wild-type tumors, PIK3CA exon 9 mutations had no effect, whereas exon 20 mutations were associated with poor clinical response to cetuximab compared to wild-type PIK3CA tumors. It must be emphasized that exon 20 PIK3CA mutations are relatively rare in mCRC (2-3\% of the cases) and this makes difficult to confirm their predictive role within randomized clinical trials.

\section{Gene fusions}

ALK, ROS1, NTRK and RET rearrangements have been described in CRC [109-113]. Although these genetic alterations are present in CRC at a quite low frequency $(<1 \%)$, they seem more frequent in CRC patients with microsatellite instability.

Gene fusions can represent important targets for therapeutic intervention. In particular, NTRK inhibitors have demonstrated clinical activity in patients with gene rearrangements that result in fusions of NTRK 1, 2 and 3 with different partners [114]. The activity of NTRK inhibitors has been demonstrated in patients with NTRK fusions regardless of the histological type and the FDA and the European Medicine Agency have subsequently approved for the first time NTRK fusions as an agnostic marker (independent of histology) for a target therapy (Table 1).

\section{NF1}

The NF1 gene encodes for neurofibromin 1, which functions as a negative regulator of KRAS [115]. Previous studies demonstrated that inactivation of NF1 leads to resistance to EGFR tyrosine kinase inhibitors in lung cancer [116]. In a recent study of cetuximab-based therapy in a small cohort of Chinese mCRC patients, NF1 mutations were associated with the shortest PFS [117]. In agreement with these findings, analyses of cohorts of mCRC patients who did not benefit from cetuximab treatment identified NF1 mutations as possible mechanisms of intrinsic resistance to anti-EGFR monoclonal antibodies $[118,119]$. Functional studies in CRC cells in which the NF1 gene was inactivated confirmed the potential role in resistance to anti-EGFR monoclonal antibodies. Further studies are definitely needed to confirm these preliminary findings (Table 1).

\section{MAP2K1}

Mutations of the MAP2K1 gene coding for MEK1 have been consistently found to be associated with de novo and acquired resistance to anti-EGFR agents in different studies [118-121]. Mechanistically, these variants lead to constitutive activation of MEK1 and increased downstream signaling. Although activating mutations of the catalytic site of MEK1 are quite rare in CRC, their identification might lead to a better identification of patients with primary resistance to anti-EGFR monoclonal antibodies. 
POLE

The POLE gene encodes for the proofreading exonuclease domain of polymerase epsilon [122]. Pathogenic somatic POLE mutations occur in approximately $1.0 \%$ of CRCs. The patients who harbor these mutations have hypermutated tumors, which are expected to carry increased neoantigen load that may predict a response to immunotherapy $[6,123]$. In a retrospective analysis of more than 4,500 patients with stage II/ III CRC, the presence of POLE mutations identified a subset of CRC patients with favorable prognosis [123]. Due to the very good prognosis of POLE mutant CRC, we might expect that a very limited fraction of mCRC patients carry mutations in POLE.

\section{Consensus molecular subtypes}

Different classification of CRC based on gene expression profiling have been proposed [124-128]. In order to solve inconsistencies among the reported gene expression-based classifications and facilitate clinical translation, the International Colorectal Cancer Consortium proposed a classification of CRC into four distinct consensus molecular subtypes (CMS) [129]. CMS-1, the MSI immune, represents $14 \%$ of all CRC and shows higher rates of MSI, CpG island methylator phenotype high, hypermutation, and BRAF mutations. The CMS-2, canonical subtype, is found in $37 \%$ of CRC, and is associated with higher rates of somatic copy number alterations and WNT and MYC pathways activation. The CMS-3 metabolic subtype represents $13 \%$ of CRC. This group is characterized by lower rates of copy number alterations, high rates of KRAS mutations and metabolic dysregulation. The CMS-4 mesenchymal subtype including $23 \%$ of CRC shows high rates of transforming growth factor- $\beta$ activation and angiogenesis. Finally, 13\% of CRC cases have mixed features and they possibly represent a transition phenotype or intra-tumor heterogeneity [129].

The CMS classification has a prognostic value. In fact, the CMS-4 CRC group has the worse OS $[129,130]$. However, the CMS-1 subtype shows the worse survival after relapse. This observation is consistent with the poor outcome of MSI-H mCRC patients.

The CALGB/SWOG 80405 and FIRE-3 studies explored the correlation between CMS classification and activity of cetuximab- and bevacizumab-based first line therapy in mCRC patients [131, 132] (Table 2). Quite surprisingly, the two studies reported quite different conclusions. While in the CALGB/SWOG 80405 trial, cetuximab treatment was correlated with a longer OS as compared with bevacizumab in the CMS-2 and CMS-3 subgroups, in the FIRE 3 study cetuximab did better than becvacizumab in all subgroups but CMS3. Several factors, including the different chemotherapy backbone of the two trials and the use of different methods for CMS classification, might have caused such discrepancy [133]. Other studies confirmed that the CMS-2 CRC subgroup is likely the most sensitive to anti-EGFR agents [119]. However, patients in subgroups other than CMS-2 might respond to anti-EGFR monoclonal antibodies, and some CMS-2 CRC might be refractory to anti-EGFR based-therapy [119]. Taken together, these findings suggest that the predictive value of the CMS classification needs further evaluation in prospective clinical trials.

\section{Conclusions and future perspectives}

While international guidelines suggest testing mCRC patients for KRAS, NRAS, BRAF and MSI only, the evidence presented in this review suggests that many other biomarkers may have an important role in personalizing treatments in mCRC. In particular, some genetic alterations may represent mechanisms of primary resistance to anti-EGFR agents but above all offer the possibility of targeted therapy. For example, tumors carrying genetic alterations in the MAPK pathway such as MAP2K1 or NF1 mutations might benefit treatment with combinations of MEK, BRAF and/or anti-EGFR agents (Table 1). A consideration that needs to be made is that many of the alterations discussed are relatively rare in mCRC. This poses a problem in the clinical validation of their prognostic role, which should be performed in randomized clinical trials. One possible solution could be to group all the alterations in a single panel, as recently proposed [134]. However, we must admit that each genetic alteration could have a different biological role.

The classification of CRC based on its driver mutations revealed the presence of a remarkable intertumor heterogeneity. To this phenomenon, however, is added that of intra-tumor heterogeneity. Numerous 
studies have shown that tumors, including CRC, often contain several clones. In this regard, multiple driver mutations, sometime at different allelic frequencies, are often identified in CRC [107]. Low-frequency allelic mutations may play a role in primary or acquired resistance to targeted therapies. For example, $R A S$ mutations at low allelic frequency have been detected in some CRC patients who demonstrated resistance to anti-EGFR drugs $[27,28,134]$. It is possible that these mutations are selected during the neoplastic progression and the treatments received by the patient. Indeed, evidence suggests that tumor heterogeneity increases significantly during treatment, favoring the appearance of resistant clones. In this regard, the majority of the mechanisms involved in primary resistance to anti-EGFR drugs play also a role in acquired resistance. On the other hand, genetic alterations at low allelic frequency might represent sub-optimal targets for therapeutic intervention. Therefore, information on the clonality of the identified mutations should be included in the referral and discussed in the molecular tumor board for treatment decision.

In this scenario, liquid biopsy and in particular the testing of circulating cell-free DNA (cfDNA) might allow to better recapitulate the heterogeneity of $\mathrm{MCRC}$ and provide relevant information for the clinical management of CRC patients. Indeed, we and other groups found that cfDNA testing allows to identify RAS mutations that are at low allelic frequency in the primary tumors but that are possibly enriched in metastatic sites $[27,28]$. Tracking genetic alterations in the cfDNA might also allow monitoring the response to treatment and the molecular evolution of the disease [135]. The development of high sensitive NGS-based methods for cfDNA testing is expanding the use of this approach also to early diagnosis and detection of minimal residual disease.

In the age of immunological therapy, many efforts have been made to optimize this approach in CRC with hitherto disappointing results overall. With the exception of MSI tumors, the majority of CRC presents characteristics of immune desert or immune tolerance. Novel approaches are definitely required in this context. In this respect, it is important to underline that also MSI tumors seem to be an heterogeneous group of tumors with some case showing an immunological background similar to MSS CRC [136].

The progress of precision medicine in the CRC requires some fundamental steps. First, it appears necessary to carry out an overall genomic and molecular profiling of CRC, which takes into account not only the genetic alterations but also the gene expression profiles and the characteristics of the tumor microenvironment. Furthermore, it will be necessary to develop algorithms to integrate all this information in order to identify the best therapeutic strategy for each individual patient. Only this approach will guarantee the application of precision medicine to the majority of patients with CRC.

\section{Abbreviations}

cfDNA: circulating cell-free DNA

CI: confidence interval

CMS: consensus molecular subtypes

COX2: cyclooxygenase- 2

CRC: colorectal cancer

dMMR: mismatch repair-deficient

EGFR: epidermal growth factor receptor

ESMO: European Society of Medical Oncology

FDA: Food and Drug Administration

HR: hazard ratio

MAPK: mitogen-activated protein-kinase

mCRC: metastatic colorectal cancer

MMR: mismatch repair

mOS: middle overall survival

MSI: microsatellite instability

MSI-H: microsatellite instability-high

MSI-L: microsatellite instability-low 
NGS: next-generation sequencing

ORR: objective response rate

OS: overall survival

PCR: polymerase chain reaction

PFS: progression free survival

PI3K: phosphatidylinositol-3-kinases

pMMR: mismatch repair-proficient

TMB: tumor mutation burden

VEGF: vascular endothelial growth factor

\section{Declarations}

\section{Author contributions}

AMR and NN contributed conception and design of the review; AMR wrote the first draft of the manuscript. All authors contributed to manuscript revision, read and approved the submitted version.

\section{Conflicts of interest}

The authors declare that they have no conflicts of interest.

\section{Ethical approval}

Not applicable.

\section{Consent to participate}

Not applicable.

\section{Consent to publication}

Not applicable.

\section{Availability of data and materials}

Not applicable.

\section{Funding}

Not applicable.

\section{Copyright}

(C) The Author(s) 2020.

\section{References}

1. Parkin DM, Bray F, Ferlay J, Pisani P. Global cancer statistics, 2002. CA Cancer J Clin. 2005;55:74-108.

2. Wolpin BM, Mayer RJ. Systemic treatment of colorectal cancer. Gastroenterology. 2008;134:1296-310.

3. Boeckx N, Janssens K, Van Camp G, Rasschaert M, Papadimitriou K, Peeters M, et al. The predictive value of primary tumor location in patients with metastatic colorectal cancer: a systematic review. Crit Rev Oncol Hematol. 2018;121:1-10.

4. Van Cutsem E, Cervantes A, Adam R, Sobrero A, Van Krieken JH, Aderka D, et al. ESMO consensus guidelines for the management of patients with metastatic colorectal cancer. Ann Oncol. 2016;27:1386-422.

5. Normanno N, Tejpar S, Morgillo F, De Luca A, Van Cutsem E, Ciardiello F. Implications for KRAS status and EGFR-targeted therapies in metastatic CRC. Nat Rev Clin Oncol. 2009;6:519-27.

6. The Cancer Genome Atlas Network. Comprehensive molecular characterization of human colon and rectal cancer. Nature. 2012;48:330-7.

7. Goodsell DS. The molecular perspective: the ras oncogene. Oncologist. 1999;4:263-4.

8. Cox AD, Fesik SW, Kimmelman AC, Luo J, Der CJ. Drugging the undruggable RAS: mission possible? Nat 
Rev Drug Discov. 2014;13:828-51.

9. Piton N, Lonchamp E, Nowak F, Sabourin JC; KRAS group. Real-life distribution of KRAS and NRAS mutations in metastatic colorectal carcinoma from French routine genotyping. Cancer Epidemiol Biomarkers Prev. 2015;24:1416-8.

10. Amado RG, Wolf M, Peeters M, Van Cutsem E, Siena S, Freeman DJ, et al. Wild-type KRAS is required for panitumumab efficacy in patients with metastatic colorectal cancer. J Clin Oncol. 2008;26:1626-34.

11. Karapetis CS, Khambata-Ford S, Jonker DJ, O'Callaghan CJ, Tu D, Tebbutt NC, et al. K-ras mutations and benefit from cetuximab in advanced colorectal cancer. N Engl J Med. 2008;359:1757-65.

12. Bokemeyer C, Bondarenko I, Makhson A, Hartmann JT, Aparicio J, de Braud F, et al. Fluorouracil, leucovorin, and oxaliplatin with and without cetuximab in the first-line treatment of metastatic colorectal cancer. J Clin Oncol. 2009;27:663-71.

13. Van Cutsem E, Köhne CH, Hitre E, Zaluski J, Chang Chien CR, Makhson A, et al. Cetuximab and chemotherapy as initial treatment for metastatic colorectal cancer. N Engl J Med. 2009;360:1408-17.

14. Peeters M, Price TJ, Cervantes A, Sobrero AF, Ducreux M, Hotko Y, et al. Randomized phase III study of panitumumab with fluorouracil, leucovorin, and irinotecan (FOLFIRI) compared with FOLFIRI alone as second-line treatment in patients with metastatic colorectal cancer. J Clin Oncol. 2010;28:4706-13.

15. Douillard JY, Siena S, Cassidy J, Tabernero J, Burkes R, Barugel M, et al. Randomized, phase III trial of panitumumab with infusional fluorouracil, leucovorin, and oxaliplatin (FOLFOX4) versus FOLFOX4 alone as first-line treatment in patients with previously untreated metastatic colorectal cancer: the PRIME study. J Clin Oncol. 2010;28:4697-705.

16. Bokemeyer C, Bondarenko I, Hartmann JT, de Braud F, Schuch G, Zubel A, et al. Efficacy according to biomarker status of cetuximab plus FOLFOX-4 as first-line treatment for metastatic colorectal cancer: the OPUS study. Ann Oncol. 2011;22:1535-46.

17. Maughan TS, Adams RA, Smith CG, Meade AM, Seymour MT, Wilson RH, et al; MRC COIN Trial Investigators. Addition of cetuximab to oxaliplatin-based first-line combination chemotherapy for treatment of advanced colorectal cancer: results of the randomised phase 3 MRC COIN trial. Lancet. 2011;377:2103-14.

18. Sorich MJ, Wiese MD, Rowland A, Kichenadasse G, McKinnon RA, Karapetis CS. Extended RAS mutations and anti-EGFR monoclonal antibody survival benefit in metastatic colorectal cancer: a meta-analysis of randomized, controlled trials. Ann Oncol. 2015;26:13-21.

19. Bokemeyer C, Kohne CH, Ciardiello F, Lenz HJ, Heinemann V, Klinkhardt U, et al. FOLFOX4 plus cetuximab treatment and RAS mutations in colorectal cancer. Eur J Cancer. 2015;51:1243-52.

20. Van Cutsem E, Lenz HJ, Kohne CH, Heinemann V, Tejpar S, Melezínek I, et al. Fluorouracil, leucovorin, and irinotecan plus cetuximab treatment and RAS mutations in colorectal cancer. J Clin Oncol. 2015;33:692-700.

21. Douillard JY, Oliner KS, Siena S, Tabernero J, Burkes R, Barugel M, et al. Panitumumab-FOLFOX4 treatment and RAS mutations in colorectal cancer. N Engl J Med. 2013;369:1023-34.

22. Peeters M, Oliner KS, Price TJ, Cervantes A, Sobrero AF, Ducreux M, et al. Analysis of KRAS/NRAS mutations in a phase III study of panitumumab with FOLFIRI compared with FOLFIRI alone as secondline treatment for metastatic colorectal cancer. Clin Cancer Res. 2015;21:5469-79.

23. Stintzing S, Modest DP, Rossius L, Lerch MM, von Weikersthal LF, Decker T, et al. FOLFIRI plus cetuximab versus FOLFIRI plus bevacizumab for metastatic colorectal cancer (FIRE-3): a post-hoc analysis of tumour dynamics in the final RAS wild-type subgroup of this randomised open-label phase 3 trial. Lancet Oncol. 2016;17:1426-34.

24. Sepulveda AR, Hamilton SR, Allegra CJ, Grody W, Cushman-Vokoun AM, Funkhouser WK, et al. Molecular biomarkers for the evaluation of colorectal cancer: guideline from the American Society for Clinical Pathology, College of American Pathologists, Association for Molecular Pathology, and American Society of Clinical Oncology. J Mol Diagn. 2017;19:187-225.

25. McGranahan N, Favero F, de Bruin EC, Birkbak NJ, Szallasi Z, Swanton C. Clonal status of actionable driver events and the timing of mutational processes in cancer evolution. Sci Transl Med. 
2015;7:283ra54.

26. Normanno N, Rachiglio AM, Lambiase M, Martinelli E, Fenizia F, Esposito C, et al. Heterogeneity of KRAS, NRAS, BRAF and PIK3CA mutations in metastatic colorectal cancer and potential effects on therapy in the CAPRI GOIM trial. Ann Oncol. 2015;26:1710-4.

27. Normanno N, Esposito Abate R, Lambiase M, Forgione L, Cardone $C$, Iannaccone A, et al. RAS testing of liquid biopsy correlates with the outcome of metastatic colorectal cancer patients treated with firstline FOLFIRI plus cetuximab in the CAPRI-GOIM trial. Ann Oncol. 2018;29:112-8.

28. Khan KH, Cunningham D, Werner B, Vlachogiannis G, Spiteri I, Heide T, et al. Longitudinal liquid biopsy and mathematical modeling of clonal evolution forecast time to treatment failure in the PROSPECT-C phase II colorectal cancer clinical trial. Cancer Discov. 2018;8:1270-85.

29. Vidal J, Bellosillo B, Santos Vivas C, García-Alfonso P, Carrato A, Cano MT, et al. Ultra-selection of metastatic colorectal cancer patients using next-generation sequencing to improve clinical efficacy of anti-EGFR therapy. Ann Oncol. 2019;30:439-46.

30. Santos C, Azuara D, Viéitez JM, Páez D, Falcó E, Élez E, et al. Phase II study of high-sensitivity genotyping of KRAS, NRAS, BRAF and PIK3CA to ultra-select metastatic colorectal cancer patients for panitumumab plus FOLFIRI: the ULTRA trial. Ann Oncol. 2019;30:796-803.

31. O'Bryan JP. Pharmacological targeting of RAS: recent success with direct inhibitors. Pharmacol Res. 2019;139:503-11.

32. Hallin J, Engstrom LD, Hargis L, Calinisan A, Aranda R, Briere DM, et al.The KRASG12C inhibitor, MRTX849, provides insight toward therapeutic susceptibility of KRAS mutant cancers in mouse models and patients. Cancer Discov. 2020;10:54-71.

33. Papadopoulos KP, Ou SI, Johnson ML, Christensen J, Velastegui K, Potvin D, et al. A phase I/II multiple expansion cohort trial of MRTX849 in patients with advanced solid tumors with KRAS G12C mutation. J Clin Oncol. 2019;37:15_suppl TPS3161.

34. Canon J, Rex K, Saiki AY, Mohr C, Cooke K, Bagal D, et al. The clinical KRAS(G12C) inhibitor AMG 510 drives anti-tumour immunity. Nature. 2019;575:217-23.

35. Govindan R, Fakih MG, Price TJ, Falchook GS, Desai J, Kuo JC, et al. Phase I study of AMG 510, a novel molecule targeting KRAS G12C mutant solid tumours. Annn Oncol. 2019;30 suppl_5:v163-4.

36. Yuan ZX, Wang XY, Qin QY, Chen DF, Zhong QH, Wang L, et al. The prognostic role of BRAF mutation in metastatic colorectal cancer receiving anti-EGFR monoclonal antibodies: a meta-analysis. PLoS One. 2013;8:e65995.

37. Forbes SA, Bhamra G, Bamford S, Dawson E, Kok C, Clements J, et al. The catalogue of somatic mutations in cancer (COSMIC). Curr Protoc Hum Genet. 2008;57.

38. Wan PT, Garnett MJ, Roe SM, Lee S, Niculescu-Duvaz D, Good VM, et al. Mechanism of activation of the RAF-ERK signaling pathway by oncogenic mutations of B-RAF. Cell. 2004;116:855-67.

39. Davies H, Bignell GR, Cox C, Stephens P, Edkins S, Clegg S, et al. Mutations of the BRAF gene in human cancer. Nature. 2002;417:949-54.

40. Jass JR. Classification of colorectal cancer based on correlation of clinical, morphological and molecular features. Histopathology. 2007;50:113-30.

41. Schirripa M, Lenz HJ. Biomarker in colorectal cancer. Cancer J. 2016;22:156-64.

42. Lai E, Pretta A, Impera V, Mariani S, Giampieri R, Casula L, et al. BRAF-mutant colorectal cancer, a different breed evolving. Expert Rev Mol Diagn. 2018;18:499-512.

43. Roma C, Rachiglio AM, Pasquale R, Fenizia F, Iannaccone A, Tatangelo F, et al. BRAF V600E mutation in metastatic colorectal cancer: Methods of detection and correlation with clinical and pathologic features. Cancer Biol Ther. 2016;17:840-8.

44. Tran B, Kopetz S, Tie J, Gibbs P, Jiang ZQ, Lieu CH, et al. Impact of BRAF mutation and microsatellite instability on the pattern of metastatic spread and prognosis in metastatic colorectal cancer. Cancer. 2011;117:4623-32.

45. Fariña-Sarasqueta A, van Lijnschoten G, Moerland E, Creemers GJ, Lemmens VE, Rutten HJ, et al. The BRAF V600E mutation is an independent prognostic factor for survival in stage II and stage III colon 
cancer patients. Ann Oncol. 2010;21:2396-402.

46. Tol J, Nagtegaal ID, Punt CJ. BRAF mutation in metastatic colorectal cancer. N Engl J Med. 2009;361:98-9.

47. Sinicrope FA, Shi Q, Allegra CJ, Smyrk TC, Thibodeau SN, Goldberg RM, et al. Association of DNA mismatch repair and mutations in BRAF and KRAS with survival after recurrence in stage III colon cancers: a secondary analysis of 2 randomized clinical trials. JAMA Oncol. 2017;3:472-80.

48. Teng HW, Huang YC, Lin JK, Chen WS, Lin TC, Jiang JK, et al. BRAF mutation is a prognostic biomarker for colorectal liver metastasectomy. J Surg Oncol. 2012;106:123-9.

49. Karagkounis G, Torbenson MS, Daniel HD, Azad NS, Diaz LA Jr, Donehower RC, et al. Incidence and prognostic impact of KRAS and BRAF mutation in patients undergoing liver surgery for colorectal metastases. Cancer. 2013;119:4137-44.

50. Schirripa M, Bergamo F, Cremolini C, Casagrande M, Lonardi S, Aprile G, et al. BRAF and RAS mutations as prognostic factors in metastatic colorectal cancer patients undergoing liver resection. Br J Cancer. 2015;112:1921-8.

51. Seligmann JF, Fisher D, Smith CG, Richman SD, Elliott F, Brown S, et al. Investigating the poor outcomes of BRAF-mutant advanced colorectal cancer: analysis from 2530 patients in randomised clinical trials. Ann Oncol. 2017;28:562-8.

52. Yao Z, Torres NM, Tao A, Gao Y, Luo L, Li Q, et al. BRAF mutants evade ERK-dependent feedback by different mechanisms that determine their sensitivity to pharmacologic inhibition. Cancer Cell. 2015;28:370-83.

53. Yao Z, Yaeger R, Rodrik-Outmezguine VS, Tao A, Torres NM, Chang MT, et al. Tumours with class 3 BRAF mutants are sensitive to the inhibition of activated RAS. Nature. 2017;548:234-8.

54. Jones JC, Renfro LA, Al-Shamsi HO, Schrock AB, Rankin A, Zhang BY, et al. Non-V600BRAF mutations define a clinically distinct molecular subtype of metastatic colorectal cancer. J Clin Oncol. 2017;35:2624-30.

55. Cremolini C, Di Bartolomeo M, Amatu A, Antoniotti C, Moretto R, Berenato R, et al. BRAF codons 594 and 596 mutations identify a new molecular subtype of metastatic colorectal cancer at favorable prognosis. Ann Oncol. 2015;26:2092-7.

56. Schirripa M, Biason P, Lonardi S, Pella N, Pino MS, Urbano F, et al. Class 1, 2, and 3 BRAF-mutated metastatic colorectal cancer: a detailed clinical, pathologic, and molecular characterization. Clin Cancer Res. 2019;25:3954-61.

57. Pietrantonio F, Petrelli F, Coinu A, Di Bartolomeo M, Borgonovo K, Maggi C, et al. Predictive role of BRAF mutations in patients with advanced colorectal cancer receiving cetuximab and panitumumab: a metaanalysis. Eur J Cancer. 2015;51:587-94.

58. Rowland A, Dias MM, Wiese MD, Kichenadasse G, McKinnon RA, Karapetis CS, et al. Meta-analysis of BRAF mutation as a predictive biomarker of benefit from anti-EGFR monoclonal antibody therapy for RAS wild-type metastatic colorectal cancer. Br J Cancer. 2015;112:1888-94.

59. Prahallad A, Sun C, Huang S, Di Nicolantonio F, Salazar R, Zecchin D, et al. Unresponsiveness of colon cancer to BRAF (V600E) inhibition through feedback activation of EGFR. Nature. 2012;483:100-3.

60. Corcoran RB, Atreya CE, Falchook GS, Kwak EL, Ryan DP, Bendell JC, et al. Combined BRAF and MEK inhibition with dabrafenib and trametinib in BRAF V600-mutant colorectal cancer. J Clin Oncol. 2015;33:4023-31.

61. Corcoran RB, André T, Atreya CE, Schellens JHM, Yoshino T, Bendell JC, et al. Combined BRAF, EGFR, and MEK inhibition in patients with BRAFV600E-mutant colorectal cancer. Cancer Discov. 2018;8:428-43.

62. Kopetz S, McDonough SL, Morris VK, Lenz HJ, Magliocco AM, Atreya CE, et al. Randomized trial of irinotecan and cetuximab with or without vemurafenib in BRAF-mutant metastatic colorectal cancer (SWOG 1406). J Clin Oncol. 2017;35:520.

63. Kopetz S, Grothey A, Yaeger R, Van Cutsem E, Desai J, Yoshino T, et al. Encorafenib, binimetinib, and cetuximab in BRAF V600E-mutated colorectal cancer. N Engl J Med. 2019;381:1632-43.

64. Zhang L. Immunohistochemistry versus microsatellite instability testing for screening colorectal cancer patients at risk for hereditary nonpolyposis colorectal cancer syndrome. Part II. The utility of 
microsatellite instability testing. J Mol Diagn. 2008;10:301-7.

65. Ward R, Meagher A, Tomlinson I, O'Connor T, Norrie M, Wu R, et al. Microsatellite instability and the clinicopathological features of sporadic colorectal cancer. Gut. 2001;48:821-9.

66. Graham DM, Coyle VM, Kennedy RD, Wilson RH. Molecular subtypes and personalized therapy in metastatic colorectal cancer. Curr Colorectal Cancer Rep. 2016;12:141-50.

67. Ashktorab H, Ahuja S, Kannan L, Llor X, Ellis NA, Xicola RM, et al. A meta-analysis of MSI frequency and race in colorectal cancer. Oncotarget. 2016;7:34546-57.

68. Luchini C, Bibeau F, Ligtenberg MJL, Singh N, Nottegar A, Bosse T, et al. ESMO recommendations on microsatellite instability testing for immunotherapy in cancer, and its relationship with PD-1/PDL1 expression and tumour mutational burden: a systematic review-based approach. Ann Oncol. 2019;30:1232-43.

69. Guastadisegni C, Colafranceschi M, Ottini L, Dogliotti E. Microsatellite instability as a marker of prognosis and response to therapy: a meta-analysis of colorectal cancer survival data. Eur J Cancer. 2010;46:2788-98.

70. Schumacher TN, Schreiber RD. Neoantigens in cancer immunotherapy. Science. 2015;348:69-74.

71. Le DT, Durham JN, Wang H, Bartlett BR, Kemberling H, Eyring AD, et al. PD-1 blockade in tumors with mismatch-repair deficiency. N Engl J Med. 2015;372:2509-20.

72. Le DT, Durham JN, Smith KN, Wang H, Bartlett BR, Aulakh LK, et al. Mismatch repair deficiency predicts response of solid tumors to PD-1 blockade. Science. 2017;357:409-13.

73. Overman MJ, McDermott R, Leach JL, Lonardi S, Lenz HJ, Morse MA, et al. Nivolumab in patients with metastatic DNA mismatch repair-deficient or microsatellite instability-high colorectal cancer (CheckMate 142): an open-label, multicentre, phase 2 study. Lancet Oncol. 2017;18:1182-91.

74. Fabrizio DA, George TJ Jr, Dunne RF, Frampton G, Sun J, Gowen K, et al. Beyond microsatellite testing: assessment of tumor mutational burden identifies subsets of colorectal cancer who may respond to immune checkpoint inhibition. J Gastrointest Oncol. 2018;9:610-7.

75. Schrock AB, Ouyang C, Sandhu J, Sokol E, Jin D, Ross JS, et al. Tumor mutational burden is predictive of response to immune checkpoint inhibitors in MSI-high metastatic colorectal cancer. Ann Oncol. 2019;30:1096-103.

76. Siena S, Sartore-Bianchi A, Marsoni S, Hurwitz HI, McCall SJ, Penault-Llorca F, et al. Targeting the human epidermal growth factor receptor 2 (HER2) oncogene in colorectal cancer. Ann Oncol. 2018;29:1108-19.

77. Ross JS, Fakih M, Ali SM, Elvin JA, Schrock AB, Suh J, et al. Targeting HER2 in colorectal cancer: the landscape of amplification and short variant mutations in ERBB2 and ERBB3. Cancer. 2018;124:1358-73.

78. Seo AN, Kwak Y, Kim DW, Kang SB, Choe G, Kim WH, et al. Her2 status in colorectal cancer: its clinical significance and the relationship between HER2 gene amplification and expression. PLoS One. 2014; 9:e98528.

79. Ingold Heppner B, Behrens HM, Balschun K, Haag J, Krüger S, Becker T, et al. HER2/neu testing in primary colorectal carcinoma. Br J Cancer. 2014;111:1977-84.

80. Missiaglia E, Jacobs B, D’Ario G, Di Narzo AF, Soneson C, Budinska E, et al. Distal and proximal colon cancers differ in terms of molecular, pathological, and clinical features. Ann Oncol. 2014;25:1995-2001.

81. Sartore-Bianchi A, Trusolino L, Martino C, Bencardino K, Lonardi S, Bergamo F, et al. Dual-targeted therapy with trastuzumab and lapatinib in treatment-refractory, KRAS codon 12/13 wild-type, HER2positive metastatic colorectal cancer (HERACLES): a proof-of-concept, multicentre, open-label, phase 2 trial. Lancet Oncol. 2016;17:738-46.

82. Raghav KPS, Overman MJ, Yu R, Meric-Bernstam F, Menter D, Kee BK, et al. HER2 amplification as a negative predictor biomarker for anti-epidermal growth factor receptor antibody therapy in metastatic colorectal cancer. J Clin Oncol. 2016;34:15_suppl 3517.

83. Yonesaka K, Zejnullahu K, Okamoto I, Satoh T, Cappuzzo F, Souglakos J, et al. Activation of ERBB2 signaling causes resistance to the EGFR-directed therapeutic antibody cetuximab. Sci Transl Med. 2011;3:99ra86.

84. Martin V, Landi L, Molinari F, Fountzilas G, Geva R, Riva A, et al. HER2 gene copy number status may 
influence clinical efficacy to anti-EGFR monoclonal antibodies in metastatic colorectal cancer patients. Br J Cancer. 2013;108:668-75.

85. Sartore-Bianchi A, Amatu A, Porcu L, Ghezzi S, Lonardi S, Leone F, et al. HER2 positivity predicts unresponsiveness to EGFR-targeted treatment in metastatic colorectal cancer. Oncologist. 2019;24:1395-402.

86. Hainsworth JD, Meric-Bernstam F, Swanton C, Hurwitz H, Spigel DR, Sweeney C, et al. Targeted therapy for advanced solid tumors on the basis of molecular profiles: results from MyPathway, an open-label, phase IIa multiple basket study. J Clin Oncol. 2018;36:536-42.

87. Ramanathan RK, Hwang JJ, Zamboni WC, Sinicrope FA, Safran H, Wong MK, et al. Low overexpression of HER-2/neu in advanced colorectal cancer limits the usefulness of trastuzumab (Herceptin) and irinotecan as therapy. A phase II trial. Cancer Invest. 2004;22:858-65.

88. Clark JW, Niedzwiecki D, Hollis D, Mayer R. Phase-II trial of 5-fluororuacil (5-FU), leucovorin (LV), oxaliplatin (Ox), and trastuzumab (T) for patients with metastatic colorectal cancer (CRC) refractory to initial therapy. Onkologie. 2003;26 suppl 3:13-46.

89. Meric-Bernstam F, Hurwitz H, Raghav KPS, McWilliams RR, Fakih M, VanderWalde A, et al. Pertuzumab plus trastuzumab for HER2-amplified metastatic colorectal cancer (MyPathway): an updated report from a multicentre, open-label, phase 2a, multiple basket study. Lancet Oncol. 2019;20:518-30.

90. Siravegna G, Sartore-Bianchi A, Nagy RJ, Raghav K, Odegaard JI, Lanman RB, et al. Plasma HER2 (ERBB2) copy number predicts response to HER2-targeted therapy in metastatic colorectal cancer. Clin Cancer Res. 2019;25:3046-53.

91. Martinelli E, Troiani T, Sforza V, Martini G, Cardone C, Vitiello PP, et al. Sequential HER2 blockade as effective therapy in chemorefractory, HER2 gene-amplified, RAS wild-type, metastatic colorectal cancer: learning from a clinical case. ESMO Open. 2018;3:e000299.

92. Kavuri SM, Jain N, Galimi F, Cottino F, Leto SM, Migliardi G, et al. HER2 activating mutations are targets for colorectal cancer treatment. Cancer Discov. 2015;5:832-41.

93. Hyman DM, Piha-Paul SA, Won H, Rodon J, Saura C, Shapiro GI, et al. HER kinase inhibition in patients with HER2- and HER3-mutant cancers. Nature. 2018;554:189-94.

94. De Luca A, Maiello MR, D'Alessio A, Pergameno M, Normanno N. The RAS/RAF/MEK/ERK and the PI3K/AKT signalling pathways: role in cancer pathogenesis and implications for therapeutic approaches. Expert Opin Ther Targets. 2012;16 Suppl 2:S17-27.

95. Vivanco I, Sawyers CL. The phosphatidylinositol 3-Kinase AKT pathway in human cancer. Nat Rev Cancer. 2002;2:489-501.

96. Campbell IG, Russell SE, Choong DY, Montgomery KG, Ciavarella ML, Hooi CS, et al. Mutation of the PIK3CA gene in ovarian and breast cancer. Cancer Res. 2004;64:7678-81.

97. Samuels Y, Wang Z, Bardelli A, Silliman N, Ptak J, Szabo S, et al. High frequency of mutations of the PIK3CA gene in human cancers. Science. 2004;304:554.

98. De Roock W, Claes B, Bernasconi D, De Schutter J, Biesmans B, Fountzilas G, et al. Effects of KRAS, BRAF, NRAS, and PIK3CA mutations on the efficacy of cetuximab plus chemotherapy in chemotherapyrefractory metastatic colorectal cancer: a retrospective consortium analysis. Lancet Oncol. 2010;11:753-62.

99. Rosty C, Young JP, Walsh MD, Clendenning M, Sanderson K, Walters RJ, et al. PIK3CA activating mutation in colorectal carcinoma: associations with molecular features and survival. PLoS One. 2013;8:e65479.

100. Mei ZB, Duan CY, Li CB, Cui L, Ogino S. Prognostic role of tumor PIK3CA mutation in colorectal cancer: a systematic review and meta-analysis. Ann Oncol. 2016;27:1836-48.

101. Liao X, Lochhead P, Nishihara R, Morikawa T, Kuchiba A, Yamauchi M, et al. Aspirin use, tumor PIK3CA mutation, and colorectal-cancer survival. N Engl J Med. 2012;367:1596-606.

102. Domingo E, Church DN, Sieber O, Ramamoorthy R, Yanagisawa Y, Johnstone E, et al. Evaluation of PIK3CA mutation as a predictor of benefit from nonsteroidal anti-inflammatory drug therapy in colorectal cancer. J Clin Oncol. 2013;31:4297-305.

103. Sartore-Bianchi A, Martini M, Molinari F, Veronese S, Nichelatti M, Artale S, et al. PIK3CA mutations 
in colorectal cancer are associated with clinical resistance to EGFR-targeted monoclonal antibodies. Cancer Res. 2009;69:1851-7.

104. Ogino S, Nosho K, Kirkner GJ, Shima K, Irahara N, Kure S, et al. PIK3CA mutation is associated with poor prognosis among patients with curatively resected colon cancer. J Clin Oncol. 2009;27:1477-84.

105. Perrone F, Lampis A, Orsenigo M, Di Bartolomeo M, Gevorgyan A, Losa M, et al. PI3KCA/PTEN deregulation contributes to impaired responses to cetuximab in metastatic colorectal cancer patients. Ann Oncol. 2009;20:84-90.

106. Sood A, McClain D, Maitra R, Basu-Mallick A, Seetharam R, Kaubisch A, et al. PTEN gene expression and mutations in the PIK3CA gene as predictors of clinical benefit to anti-epidermal growth factor receptor antibody therapy in patients with KRAS wild-type metastatic colorectal cancer. Clin Colorectal Cancer. 2012;11:143-50.

107. Ciardiello F, Normanno N, Maiello E, Martinelli E, Troiani T, Pisconti S, et al. Clinical activity of FOLFIRI plus cetuximab according to extended gene mutation status by next-generation sequencing: findings from the CAPRI-GOIM trial. Ann Oncol. 2014;25:1756-61.

108. Souglakos J, Philips J, Wang R, Marwah S, Silver M, Tzardi M, et al. Prognostic and predictive value of common mutations for treatment response and survival in patients with metastatic colorectal cancer. Br J Cancer. 2009;101:465-72.

109. Pietrantonio F, Di Nicolantonio F, Schrock AB, Lee J, Tejpar S, Sartore-Bianchi A, et al. ALK, ROS1, and NTRK rearrangements in metastatic colorectal cancer. J Natl Cancer Inst. 2017;109.

110. Pietrantonio F, Di Nicolantonio F, Schrock AB, Lee J, Morano F, Fuca G, et al. RET fusions in a small subset of advanced colorectal cancers at risk of being neglected. Ann Oncol. 2018;29:1394-401.

111. Russo M, Misale S, Wei G, Siravegna G, Crisafulli G, Lazzari L, et al. Acquired resistance to the TRK inhibitor entrectinib in colorectal cancer. Cancer Discov. 2016;6:36-44.

112. Yaeger R, Shah MA, Miller VA, Kelsen JR, Wang K, Heins ZJ, et al. Genomic alterations observed in colitisassociated cancers are distinct from those found in sporadic colorectal cancers and vary by type of inflammatory bowel disease. Gastroenterology. 2016;151:278-87.

113. Kloosterman WP, Coebergh van den Braak RRJ, Pieterse M, van Roosmalen MJ, Sieuwerts AM, Stangl C, et al. A systematic analysis of oncogenic gene fusions in primary colon cancer. Cancer Res. 2017;77:3814-22.

114. Cocco E, Scaltriti M, Drilon A. NTRK fusion-positive cancers and TRK inhibitor therapy. Nat Rev Clin Oncol. 2018;15:731-47.

115. Cichowski K, Jacks T. NF1 tumor suppressor gene function: narrowing the GAP. Cell. 2001;104:593-604.

116. de Bruin EC, McGranahan N, Mitter R, Salm M, Wedge DC, Yates L, et al. Spatial and temporal diversity in genomic instability processes defines lung cancer evolution. Science. 2014;346:251-6.

117. Mei Z, Shao YW, Lin P, Cai X, Wang B, Ding Y, et al. SMAD4 and NF1 mutations as potential biomarkers for poor prognosis to cetuximab-based therapy in Chinese metastatic colorectal cancer patients. BMC Cancer. 2018;18:479.

118. Rachiglio AM, Lambiase M, Fenizia F, Roma C, Cardone C, Iannaccone A, et al. Genomic profiling of KRAS/NRAS/BRAF/PIK3CA wild-type metastatic colorectal cancer patients reveals novel mutations in genes potentially associated with resistance to anti-EGFR agents. Cancers. 2019;11:E859.

119. Woolston A, Khan K, Spain G, Barber LJ, Griffiths B, Gonzalez-Exposito R, et al. Genomic and transcriptomic determinants of therapy resistance and immune landscape evolution during anti-EGFR treatment in colorectal cancer. Cancer Cell. 2019;36:35-50.

120. Russo M, Siravegna G, Blaszkowsky LS, Corti G, Crisafulli G, Ahronian LG, et al. Tumor heterogeneity and lesion-specific response to targeted therapy in colorectal cancer. Cancer Discov. 2016;6:147-53.

121. Bertotti A, Papp E, Jones S, Adleff V, Anagnostou V, Lupo B, et al. The genomic landscape of response to EGFR blockade in colorectal cancer. Nature. 2015;526:263-7.

122. Palles C, Cazier JB, Howarth KM, Domingo E, Jones AM, Broderick P, et al. Germline mutations affecting the proofreading domains of POLE and POLD1 predispose to colorectal adenomas and carcinomas. Nat Genet. 2013;45:136-44. 
123. Domingo E, Freeman-Mills L, Rayner E, Glaire M, Briggs S, Vermeulen L, et al. Somatic POLE proofreading domain mutation, immune response, and prognosis in colorectal cancer: a retrospective, pooled biomarker study. Lancet Gastroenterol Hepatol. 2016;1:207-16.

124. Hoadley KA, Yau C, Wolf DM, Cherniack AD, Tamborero D, Ng S, et al. Multiplatform analysis of 12 cancer types reveals molecular classification within and across tissues of origin. Cell. 2014;158:929-44.

125. Budinska E, Popovici V, Tejpar S, D’Ario G, Lapique N, Sikora KO, et al. Gene expression patterns unveil a new level of molecular heterogeneity in colorectal cancer. J Pathol. 2013;231:63-76.

126. Sadanandam A, Lyssiotis CA, Homicsko K, Collisson EA, Gibb WJ, Wullschleger S, et al. A colorectal cancer classification system that associates cellular phenotype and responses to therapy. Nat Med. 2013;19:619-25.

127. Marisa L, de Reyniès A, Duval A, Selves J, Gaub MP, Vescovo L, et al. Gene expression classification of colon cancer into molecular subtypes: characterization, validation, and prognostic value. PLoS Med. 2013;10:e1001453.

128. Perez-Villamil B, Romera-Lopez A, Hernandez-Prieto S, Lopez-Campos G, Calles A, Lopez-Asenjo JA, et al. Colon cancer molecular subtypes identified by expression profiling and associated to stroma, mucinous type and different clinical behavior. BMC Cancer. 2012;12:260.

129. Guinney J, Dienstmann R, Wang X, de Reyniès A, Schlicker A, Soneson C, et al. The consensus molecular subtypes of colorectal cancer. Nat Med. 2015;21:1350-6.

130. Song N, Pogue-Geile KL, Gavin PG, Yothers G, Kim SR, Johnson NL, et al. Clinical outcome from oxaliplatin treatment in stage II/III colon cancer according to intrinsic subtypes: secondary analysis of NSABP C-07/NRG oncology randomized clinical trial. JAMA Oncol. 2016;2:1162-9.

131. Lenz HJ, Ou FS, Venook AP, Hochster HS, Niedzwiecki D, Goldberg RMJ, et al. Impact of consensus molecular subtype on survival in patients with metastatic colorectal cancer: results from CALGB/ SWOG 80405 (Alliance). J Clin Oncol. 2019;37:1876-85.

132. Stintzing S, Wirapati P, Lenz HJ, Neureiter D, Fischer von Weikersthal L, Decker T, et al. Consensus molecular subgroups (CMS) of colorectal cancer (CRC) and first-line efficacy of FOLFIRI plus cetuximab or bevacizumab: analysis of the FIRE-3 (AIO KRK 0306) study. Ann Oncol. 2019;30:1796-803.

133. Aderka D, Stintzing S, Heinemann V. Explaining the unexplainable: discrepancies in results from the CALGB/SWOG 80405 and FIRE-3 studies. Lancet Oncol. 2019;20:e274-83.

134. Morano F, Corallo S, Lonardi S, Raimondi A, Cremolini C, Rimassa L, et al. Negative hyperselection of patients with RAS and BRAF wild-type metastatic colorectal cancer who received panitumumab-based maintenance therapy. J Clin Oncol. 2019;37:3099-110.

135. Normanno N, Cervantes A, Ciardiello F, De Luca A, Pinto C. The liquid biopsy in the management of colorectal cancer patients: current applications and future scenarios. Cancer Treat Rev. 2018;70:1-8.

136. Marisa L, Svrcek M, Collura A, Becht E, Cervera P, Wanherdrick K, et al. The balance between cytotoxic T-cell lymphocytes and immune checkpoint expression in the prognosis of colon tumors. J Natl Cancer Inst. 2018;110. 